\title{
Hyposecretion of fluid from tracheal submucosal glands of CFTR-deficient pigs
}

\author{
Nam Soo Joo, Hyung-Ju Cho, Monal Khansaheb, and Jeffrey J. Wine
}

Cystic Fibrosis Research Laboratory, Stanford University, Stanford, California, USA.

\begin{abstract}
Cystic fibrosis (CF) results from mutations that disrupt CF transmembrane conductance regulator (CFTR), an anion channel found mainly in apical membranes of epithelial cells. CF leads to chronic infection of the airways with normally innocuous bacteria and fungi. Hypotheses to explain the pathophysiology of CF airways have been difficult to test because mouse models of CF do not develop human-like airway disease. The recent production of pigs lacking CFTR and pigs expressing the most common CF-causing CFTR mutant, $\Delta F 508$, provide another model that might help clarify the pathophysiology of CF airway disease. Here, we studied individual submucosal glands from 1-day-old piglets in situ in explanted tracheas, using optical methods to monitor mucus secretion rates from multiple glands in parallel. Secretion rates from control piglets (WT and $\left.C F T R^{+/-}\right)$and piglets with $\mathrm{CF}-$ like disease $\left(\mathrm{CFTR}^{-/-}\right.$and $\left.C F T R^{-/ \triangle F 508}\right)$ were measured under 5 conditions: unstimulated (to determine basal secretion), stimulated with forskolin, stimulated with carbachol, stimulated with substance $P$, and, as a test for synergy, stimulated with forskolin and a low concentration of carbachol. Glands from piglets with CF-like disease responded qualitatively to all agonists like glands from human patients with $\mathrm{CF}$, producing virtually no fluid in response to stimulation with forskolin and substantially less in response to all other agonists except carbachol. These data are a step toward determining whether gland secretory defects contribute to $\mathrm{CF}$ airway disease.
\end{abstract}

\section{Introduction}

Mucus accumulation and obstruction of exocrine glands play important roles in the pathophysiology of patients with cystic fibrosis (CF; ref. 1). Although multiple organs are involved in $\mathrm{CF}$, most people with CF die as a result of lung disease. Yet the lung is not the most severely affected organ at birth, and, when infections are held at bay, nearly normal lung function can persist for decades. This places a high premium on understanding the pathogenesis of CF lung disease, because effective postnatal intervention greatly improves the quality and length of life. Therapeutic progress has been steady, but it may be accelerated if placed on a sounder mechanistic footing.

At present, the many competing hypotheses of CF lung pathogenesis cannot be tested rigorously; for that, we need an animal model that closely mimics human pathophysiology. For this reason, CF pigs were generated (2) based on evidence that they have many features required to model human CF airway disease (3). The first studies of newborn animals suggest that CFTR ${ }^{-1}$ piglets indeed replicate many abnormalities seen in newborn humans with CF: their nasal epithelium exhibited defective chloride transport, and they showed structural evidence of exocrine pancreatic destruction, focal biliary cirrhosis, gallbladder disease, and meconium ileus (4). In a small set of pigs with CF-like disease that survived for up to 6 months after their intestinal obstruction was bypassed, clear evidence was obtained for obstruction and infection of the airways (5). Because meconium ileus in piglets with CF-like disease is severe, most of these animals must be euthanized shortly after birth, which limits longitudinal studies of pathogenesis and focuses attention on the nature of the airway innate defenses in the newborn CF-like airways. Tracheas of newborn piglets have defective

Conflict of interest: The authors have declared that no conflict of interest exists. Citation for this article: J Clin Invest. 2010;120(9):3161-3166. doi:10.1172/JCI43466. innate defenses, as indicated by a reduced ability to eradicate instilled bacteria (5). The nature of the defective innate defenses is not yet understood; altered gland secretion might play a role. In humans with $\mathrm{CF}$, the submucosal glands show profound deficiencies in fluid secretion in response to agonists that elevate intracellular cAMP concentration $\left([\mathrm{cAMP}]_{\mathrm{i}}\right)$ alone $(6)$ or with modest increases in $\left[\mathrm{Ca}^{2+}\right]_{\mathrm{i}}(7,8)$, and less profound defects in response to agonists that strongly elevate $\left[\mathrm{Ca}^{2+}\right]_{\mathrm{i}}(9-12)$. The purpose of the present experiments was to quantify the mucus secretory responses of tracheal submucosal glands in newborn control piglets and piglets with CF-like disease for comparison with one another and with glands from control and CF humans.

\section{Results}

Our primary goal was to examine fluid secretory responses of airway glands from newborn control piglets and piglets with CF-like disease, prior to any changes in the latter that might be secondary to infection and inflammation that are invariably present in the study of human CF glands. To evaluate the role of CFTR in airway gland secretion of the CF pig model, we used in situ optical monitoring to measure individual airway gland secretion rates in isolated tracheal mucosa from control piglets (WT and heterozygous $\left.\mathrm{CFTR}^{+-}\right)$and from piglets with CF-like disease $\left(C F T R^{-/ \triangle F 508}\right.$ and $\mathrm{CFTR}^{-1}$; referred to herein as CF piglets) aged about 1 day. We first measured gland density by counting the number of secreting glands in a given area in response to carbachol, the most effective agonist. The density of secreting tracheal glands from 1-day-old control and CF piglets did not differ significantly: $11 \pm 0.75$ glands $/ \mathrm{mm}^{2}$ in control piglets $\left(n=8[\mathrm{WT}] ; 2\left[\mathrm{CFTR}^{+/-}\right]\right)$and $10.1 \pm 0.9$ glands $/ \mathrm{mm}^{2}$ in CF piglets $(n=9 ; P=0.17)$.

We recognized that the reduced secretion rates of $C F$ glands might be attributed, at least in part, to a reduction in gland size. Indeed, Meyerholz et al. (13) recently estimated both gland density and gland volume in CF piglets and their WT littermates and 
A

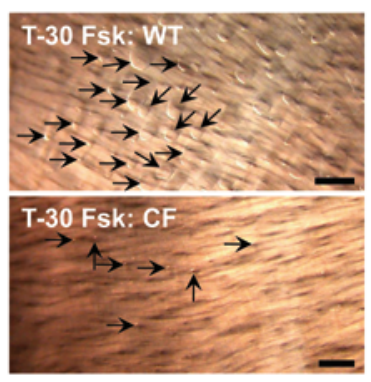

B

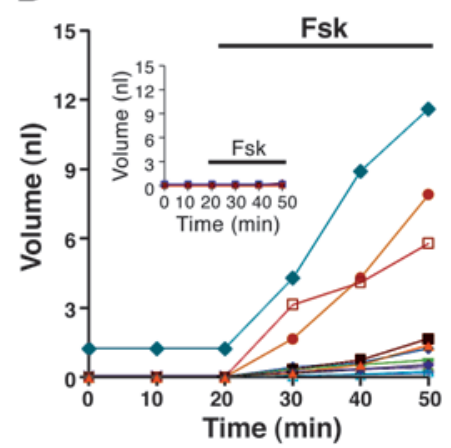

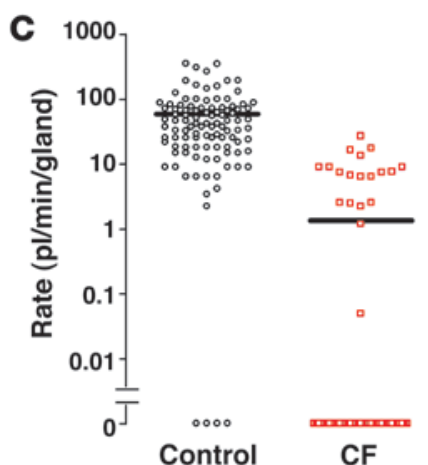

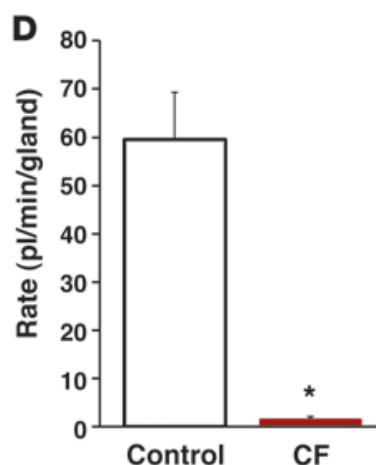

Figure 1

CF piglet glands show near-complete loss of Fsk-stimulated fluid secretion. (A) Example of mucus bubbles under oil, produced by 30 minutes' stimulation with $3 \mu \mathrm{M}$ Fsk in a WT or CF piglet trachea. Arrows denote selected mucus bubbles (19 in the WT field and 7 unambiguous bubbles in the CF field). Scale bars: $0.5 \mathrm{~mm}$. (B) Representative plot of cumulative mucus volume as a function of time and stimulation with $3 \mu \mathrm{M}$ Fsk for 12 glands in the trachea of a 1-day-old WT piglet. This is the same preparation shown in part in $\mathbf{A}$. Inset shows near-0 response of $15 \mathrm{glands}$ from a 1-day-old CF piglet. Fsk stimulation is represented by horizontal bars. (C) Secretory response rates for all glands tested with $3 \mu \mathrm{M}$ Fsk. Each symbol represents a single gland; horizontal bars denote means of 104 control (median, $37.2 \mathrm{pl} / \mathrm{min} / \mathrm{gland}$ ) and $107 \mathrm{CF}$ (median, $0 \mathrm{pl} / \mathrm{min} / \mathrm{gland}$ ) glands. Only 19 CF glands had measurable secretion in response to Fsk; 88 did not respond to Fsk, but were detected by their responses to subsequent stimulation. (D) Summary data for control and CF piglets tested with $3 \mu \mathrm{M}$ Fsk (10-15 glands each; $n=9$ per group). ${ }^{*} P=0.0002$.

found comparable gland density when counting duct openings per tracheal cross section and normalizing for the smaller tracheal circumference of the CF piglets. However, they found that submucosal gland tissue area was significantly reduced by $31 \%-37 \%$ in CF tracheal sections, depending on whether it was normalized to lumen circumference or to inner wall area (13). Thus, gland numbers are the same in CF and control piglets, but individual gland volume is reduced in CF piglets at birth. In the present study, we report observed secretion rates in picoliters per minute per gland, but also use the intermediate value of $34 \%$ reduced gland volume to correct for the response rates in CF piglets (see below).

Average basal secretion rates were less than $3 \mathrm{pl} / \mathrm{min} / \mathrm{gland}$ in all genotypes (21-197 glands; $n=2-11$ ). In human submucosal glands, the $[\mathrm{cAMP}]_{\mathrm{i}}$-elevating agonists vasoactive intestinal peptide and forskolin (Fsk) stimulate mucus secretion in control, but not $\mathrm{CF}$, tissues $(6,12)$. Figure $1 \mathrm{~A}$ shows mucus bubbles produced in tracheas of 1-day-old WT and CF piglets after 30 minutes of stimulation with $3 \mu \mathrm{M}$ Fsk. Figure 1B shows the cumulative volumes of mucus secreted from individual submucosal glands in these same tracheas. The distributions of individual control and CF gland responses to $3 \mu \mathrm{M}$ Fsk are shown in Figure 1C, and mean data for experiments with 9 control $\left(n=7\right.$ [WT]; $\left.2\left[\mathrm{CFTR}^{+/}\right]\right)$and $9 \mathrm{CF}$ piglets are shown in Figure 1D. We presented data for individual glands so that the wide range of gland secretory rates can be appreciated. However, for

\section{Figure 2}

CF piglet glands secrete less fluid in a synergy paradigm ( $3 \mu \mathrm{M}$ Fsk plus $0.1 \mu \mathrm{M}$ carbachol). (A and $\mathbf{B})$ Cumulative mucus volume as a function of time and stimulation from 12 glands of a 1-day-old WT piglet (A) and 9 glands of a 1-day-old CF piglet (B). Stimulation by the indicated agonists is represented by horizontal bars. In $\mathbf{A}$, carbachol was below threshold at 0.05 and $0.1 \mu \mathrm{M}$. (C) Secretion rates for all glands tested with $3 \mu \mathrm{M}$ Fsk plus $0.1 \mu \mathrm{M}$ carbachol. Each symbol represents a single gland; horizontal bars denote means for 101 control (median, $95.4 \mathrm{pl} / \mathrm{min} /$ gland) and $110 \mathrm{CF}$ (median, $21.4 \mathrm{pl} / \mathrm{min} / \mathrm{gland}$ ) glands. (D) Summary data for 9 control and 9 CF piglets tested with $3 \mu \mathrm{M}$ Fsk plus $0.1 \mu \mathrm{M}$ carbachol $\left(7 \mathrm{CFTR}^{-/-}\right.$and $\left.2 \mathrm{CFTR}^{-/ \Delta F 508}\right){ }^{*} P=0.007$. tests of significance, we show the average of the mean rates for each animal; gland rates within animals varied much more than average rates across animals for all species we have tested $(6,14,15)$.

Mucus secretion rates for glands stimulated with $3 \mu \mathrm{M}$ Fsk were $60.7 \pm 10.3 \mathrm{pl} / \mathrm{min} /$ gland for WT (82 glands, $n=7$ ) and $55.4 \pm 47.2$ $\mathrm{pl} / \mathrm{min} /$ gland for $\mathrm{CFTR}^{+/-}(22$ glands, $n=2)$ piglets. Because we observed no significant difference between responses in the WT and $\mathrm{CFTR}^{+/-}$piglets, we combined the results into a single control group with a mean secretion rate of $59.5 \pm 9.8 \mathrm{pl} / \mathrm{min} /$ gland $(104$ glands; $n=9)$. For CF piglets, the value was $1.5 \pm 0.6$ (107 glands, $n=9 ; P=0.00023$ ), and the size-corrected value was $1.9 \pm 0.8$ (sizecorrected $P=0.00025)$. Thus, the lack of responsiveness to Fsk of CF human glands $(6,12)$ was also seen in CF piglets.
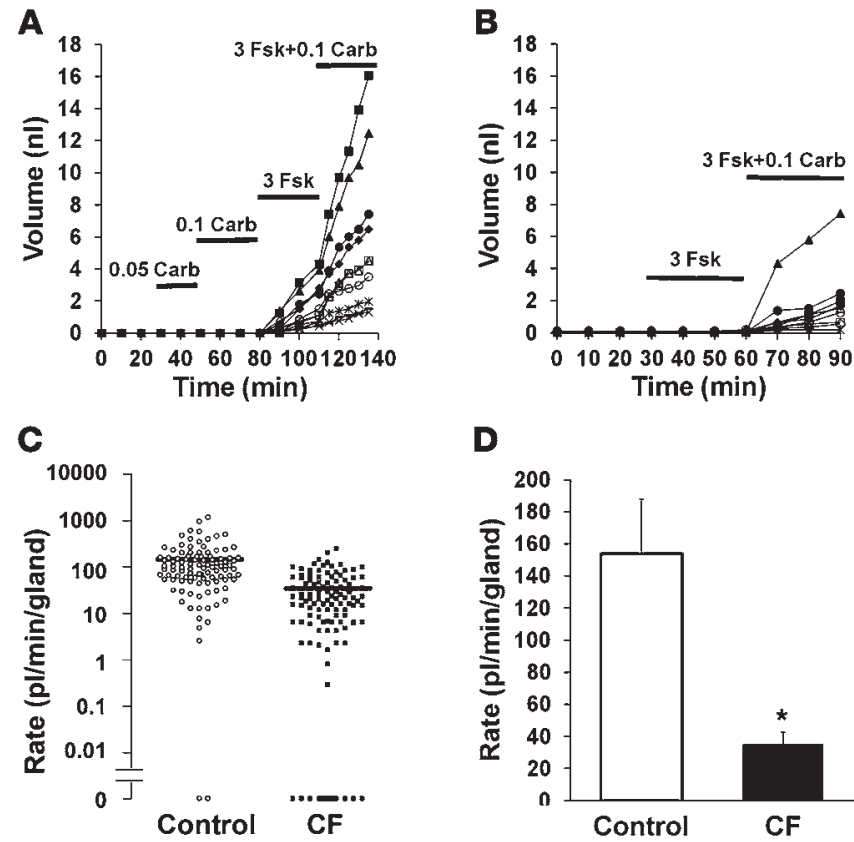

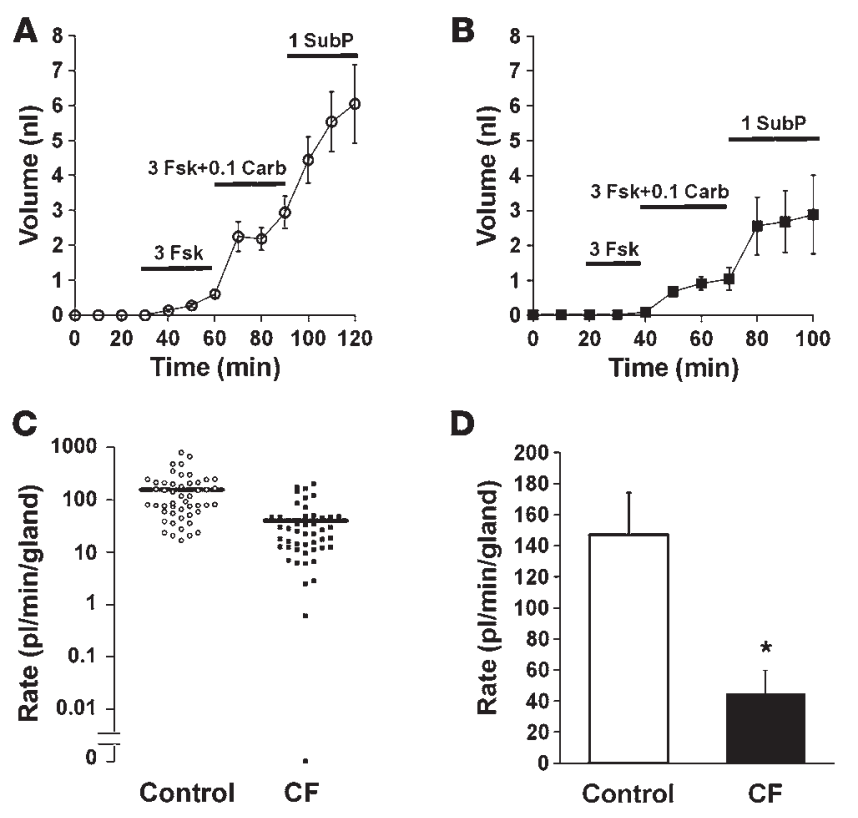

In human glands, the combination of low levels of $[\mathrm{cAMP}]_{\mathrm{i}}$ and $\left[\mathrm{Ca}^{2+}\right]_{i}$-elevating agonists produce synergistic secretion (i.e., secretion greater than the expected additive effect), but synergy was not observed in human CF glands (7). To test for synergy in the piglets, we combined $0.1 \mu \mathrm{M}$ carbachol with $3 \mu \mathrm{M}$ Fsk (Figure $2, \mathrm{~A}-\mathrm{D})$. Low concentrations of 0.05 or $0.1 \mu \mathrm{M}$ carbachol alone did not induce gland secretion, but the combined agonists simulated mean secretion of $153.9 \pm 34.1 \mathrm{pl} / \mathrm{min} /$ gland in control piglets (101 glands; $n=9$ ) compared with $35.2 \pm 7.5$ $\mathrm{pl} / \mathrm{min} /$ gland in CF piglets (110 glands, $n=9 ; P=0.0072)$, with a size-corrected value of $47.2 \pm 10.0 \mathrm{pl} / \mathrm{min} /$ gland (size-corrected $P=0.014)$. Thus, like humans $(7)$, newborn CF piglets showed a substantially smaller synergistic response to the combined agonists. However, unlike humans, the piglets did not entirely lack a synergistic response, perhaps because the level of Fsk used in the present experiments was higher than that used in humans. Because synergy was observed in CFTR-null piglets, the response must be mediated by non-CFTR pathways. The limited availability of tissues precluded a parametric examination of synergy in the CF piglets.

Substance P (SubP) elevates $\left[\mathrm{Ca}^{2+}\right]_{i}$ and stimulates secretion in both human (8) and pig glands (16), but is much more efficacious in pigs than in humans (8). In human glands, the weak secretion produced by SubP is mainly CFTR dependent, because SubP produced almost no secretion in CF glands (8). Because of these differences between humans and pigs in response to SubP, we were especially interested to study responses to SubP in CF piglets. The limited availability of tissues required us to test responses to SubP after prior exposure to Fsk and carbachol; therefore, residual effects of those agonists may have influenced the results. When tested in this way, $1 \mu \mathrm{M}$ SubP increased secretion in both controls $(147.2 \pm 27.7 \mathrm{pl} / \mathrm{min} /$ gland; 49 glands, $n=4)$ and CF piglets $(44.8 \pm 15.1 \mathrm{pl} / \mathrm{min} /$ gland; size corrected, $60 \pm 20.2 \mathrm{pl} / \mathrm{min} /$ gland; 48 glands, $n=5$ ), but secretion in CF piglets was significantly reduced to about $30 \%$ of the control response $(P=0.014)$, with a size-corrected value of $41 \%$ of controls (size-corrected $P=0.027$; Figure $3, \mathrm{~A}-\mathrm{D}$ ).

\section{Figure 3}

Responses to SubP. (A and B) Mean cumulative mucus volume from 7-9 control glands of a 1-day-old CFTR ${ }^{+/}$piglet (A) and 3-9 glands of a 1-day-old $C F T R^{-/ \triangle F 508}$ piglet (B). Stimulation by the indicated agonists is represented by horizontal bars. (C) Secretory response rates for all glands tested with $1 \mu \mathrm{M}$ SubP. Each symbol represents a single gland; horizontal bars denote means for 49 control (median, $90.1 \mathrm{pl} / \mathrm{min} /$ gland) and $48 \mathrm{CF}$ (median, $23.4 \mathrm{pl} / \mathrm{min} / \mathrm{gland}$ ) glands. All glands had been previously tested with Fsk and low-dose carbachol; the response to SubP alone was not tested in these experiments. (D) Summary data for 4 control and $5 \mathrm{CF}$ piglets tested with $1 \mu \mathrm{M}$ SubP. ${ }^{*} P=0.014$.

Although this was a significant reduction, in CF humans, secretion to SubP is reduced to near 0 (8). We hypothesize that this difference is related to the greater efficacy of SubP as a $\left[\mathrm{Ca}^{2+}\right]_{i}$-elevating secretagogue in pig glands. SubP weakly elevates $\left[\mathrm{Ca}^{2+}\right]_{\mathrm{i}}$ in human gland serous cells, where secretion to SubP is CFTR dependent (8). However, SubP is as efficacious as acetylcholine in adult pig glands (16) and approximately 10 -fold more efficacious than in human glands (8). Thus, the residual secretion to SubP in the CF piglets is probably occurring through $\mathrm{Ca}^{2+}$-activated $\mathrm{Cl}^{-}$channels (CaCCs). The reason for the greater efficacy of SubP in pigs has not been established. If the response to carbachol is taken as a standard, newborn piglets are less responsive to SubP than are older pigs. The ratio of secretion rate in response to carbachol/SubP is approximately $1: 1$ in adult pigs (17), approximately $3: 1$ in newborn piglets (present study), and 10:1 in adult humans (8). It will be interesting to see whether gland secretion in response to SubP becomes less CFTR dependent as CF piglets mature.

Acetylcholine is the most potent and efficacious agonist for gland secretion in humans and in most other species in which it has been tested. In humans with CF, cholinergic agonists produced secretion that was approximately half that of normal in young patients whose glands had not enlarged (11), whereas in the enlarged glands obtained from CF lungs at the time of transplant, secretion rates were comparable to those of healthy controls (8). In control piglets, $1 \mu \mathrm{M}$ carbachol produced secretion levels approximately 2.7 -fold those produced by $1 \mu \mathrm{M} \operatorname{SubP}(P=0.008$; Figure 3D and Figure 4D). In CF piglets, secretion to carbachol appeared to be significantly reduced to $58 \%$ of controls $(P=0.038$; Figure 4D); however, when corrected for smaller gland size in CF piglets (see below), the difference was reduced to $78 \%$ of controls and was no longer significant.

Figure 5 shows summary data with and without correction for the reduced volume of the CF glands measured by Meyerholz et al. (13). After correcting for the smaller CF gland volume, differences between secretion rates of $\mathrm{CF}$ and control piglets remained significantly different in response to all mediators except carbachol $(P=0.26)$.

The reductions in CF relative to control piglet glands were ranked by decreasing magnitude as follows: Fsk (response almost eliminated), SubP, synergy of Fsk plus carbachol, and carbachol (not significantly reduced). The order of differences was essentially the same as that for human glands, except that SubP responses in normal human glands are much smaller than in pigs and are almost eliminated in human CF glands. We cannot yet make a quantitative comparison of synergy in human and piglet glands because the paradigms differed, with a much higher concentration of Fsk being used in the experiments with piglets. 

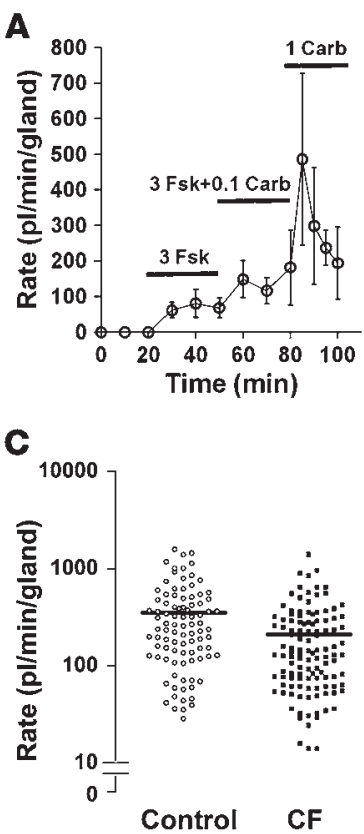

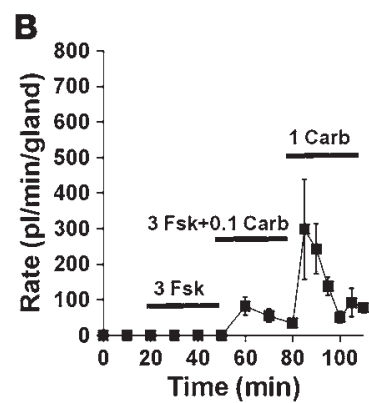

D

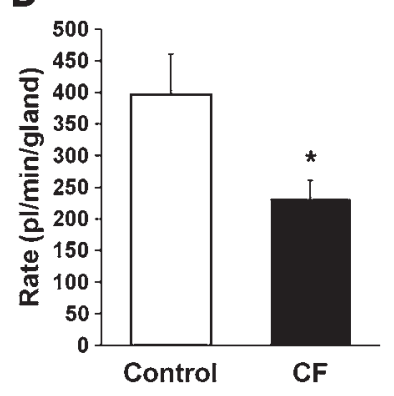

\section{Discussion}

Organ-specific consequences of CFTR mutations on fluid secretion can be attributed to the extent to which an organ depends upon CFTR or alternative pathways for anion secretion - which is sometimes, but not always related, to the stimulus pathway. Thus, in humans, CF intestinal secretion is lost to all mediators (18-20), but in airway surface epithelium and submucosal glands, CaCCs exist, at least partly constituted from TMEM16A $(21,22)$, and it is hypothesized that these can partially compensate for the loss of anion-mediated fluid secretion normally mediated by CFTR. Indeed, one current strategy to ameliorate $\mathrm{CF}$ airways disease is to accentuate secretion via these pathways (23). An important point raised by our present results is that the relative roles of these pathways appear to be similar in pig and human airway glands. This is in marked contrast to the secretory properties of mouse tracheal glands, which rely overwhelmingly on $\mathrm{CaCC}$ pathways for secretion (24).

Our results from CF piglets suggest that CFTR plays a similar role in mucus secretion from pig and human airway glands. This could be important, because innate defenses of the newborn CF tracheas are clearly defective (5). What else do we need to know before we can understand whether glands play a significant role in CF lung disease? We certainly need to know more about how gland secretion is neurally controlled. Glands are richly innervated by neurons releasing multiple transmitters, and CFTR mediates different proportions of fluid secretion depending on which pathways are active, yet we know little about how these systems operate in vivo (25). Nevertheless, it appears that fluid secretion

\section{Figure 5}

Summary data for all 5 conditions. Inset shows basal secretion rates with expanded rate axis: control (white bars), CF (black bars), and CF corrected for decreased gland volume (CF-C; gray bars). ${ }^{*} P<0.05$ vs. controls. $P$ values for size-corrected $C F$ data versus control were as follows: basal, $P=0.19$; $3 \mu \mathrm{M}$ Fsk, $P<0.0005$; synergy of $3 \mu \mathrm{M}$ Fsk and $0.1 \mu \mathrm{M}$ carbachol, $P<0.05 ; 1 \mu \mathrm{M}$ SubP, $P<0.05 ; 1 \mu \mathrm{M}$ carbachol, $P=0.26$.

\section{Figure 4}

Responses to carbachol. (A and B) Average secretion rates as a function of time and stimulation for 3 glands in the trachea of a 1-day-old WT piglet $(\mathbf{A})$ and from 8 glands in a CF piglet (B). Stimulation by the indicated agonists is represented by horizontal bars. (C) Secretory response rates for all glands tested with $1 \mu \mathrm{M}$ carbachol. Each symbol represents a single gland; horizontal bars denote means for 93 control (median, $236.2 \mathrm{pl} / \mathrm{min} / \mathrm{gland}$ ) and $119 \mathrm{CF}$ (median, 130.3 $\mathrm{pl} / \mathrm{min} / \mathrm{gland}$ ) glands. Data included glands previously tested with other agonists (controls, 51 glands, $n=6$; CF, 95 glands, $n=10$ ) as well as glands tested with $1 \mu \mathrm{M}$ carbachol alone (WT, 42 glands, $n=3$; CF, 24 glands, $n=2$ ). Responses were usually larger in both control and $\mathrm{CF}$ animals when carbachol was tested alone. (D) Summary data for 6 control and $10 \mathrm{CF}$ piglets tested with $1 \mu \mathrm{M}$ carbachol. ${ }^{*} P=0.038$.

was reduced in response to all mediators, although to different extents. We corrected for smaller gland size in order to assess the secretory potential of equivalently sized glands. However, the smaller gland size of the CF piglets will itself diminish the amount of mucus the glands secrete onto the airway surface (13), so that the uncorrected secretory values are perhaps most relevant. What are the expected consequences of diminished gland secretion?

Extensive prior work on the properties of porcine airway submucosal gland secretion has been reported by Ballard and colleagues using isolated bronchial segments (26). A theme of their studies is that when fluid secretion is decreased (as accomplished in their study by use of various anion transport inhibitors), the pig mucus becomes thicker, more tenacious, and harder to clear from glands and airways. This might hamper mechanical removal of organisms by mucus clearance. In addition, airway gland mucus contains abundant antimicrobials, and their bioavailability may be reduced if fluid secretion is not optimal $(10,27)$. This occurs in the CF mouse intestine, where microbial-containing Paneth cell granules are not dispersed, and bacterial overgrowth occurs (28). Also some antimicrobials rely specifically on CFTR for transport $(29,30)$.

To our knowledge, it has not previously been possible to test the relative importance of various potential defects of mucosal innate defenses in human CF airways. Because CF pigs develop lung infections (5), new opportunities may be available to clarify the pathogenesis of CF lung disease. It remains to be determined to what extent the defective mucus secretion from submucosal glands of CF piglets contributes to defective airway innate defenses in CF.

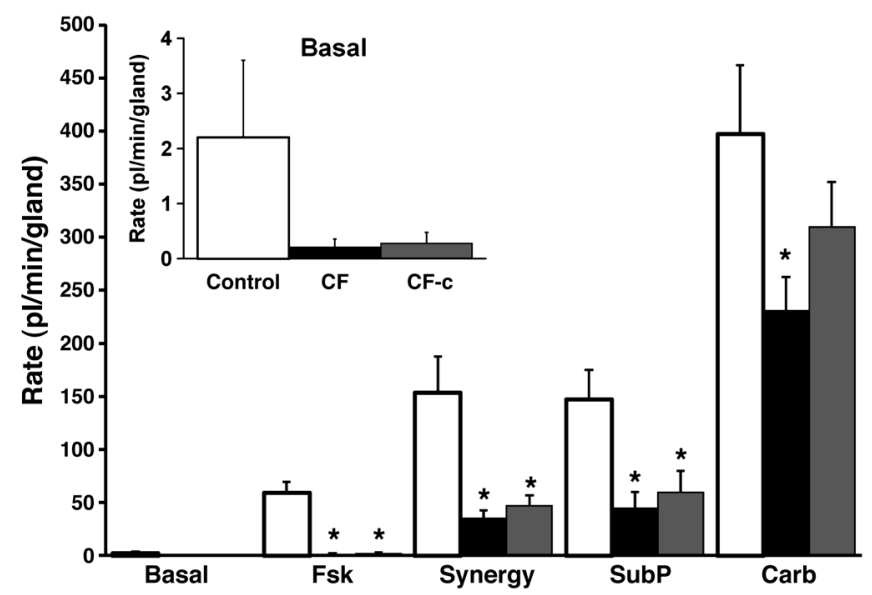




\section{Methods}

Piglet tracheas. All animal procedures were approved by the Institutional Animal Care and Use Committees of the University of Iowa (Iowa City, Iowa, USA) and Stanford University. Piglets were offspring of CFTR ${ }^{+-}$ pigs made by homologous recombination in fibroblasts from outbred domestic pigs and subsequent somatic cell nuclear transfer (2). Piglets were genotyped as described previously (4). Piglets were euthanized within 24 hours, except for $1 \mathrm{WT}$ and $1 \mathrm{CF}$ animal euthanized on day 2. The tracheas were removed, and segments of at least $0.5 \mathrm{~cm}$ length were shipped by P.H. Karp (University of Iowa, Iowa City, Iowa, USA) in cold physiological saline containing $10 \mathrm{mM}$ glucose. Some WT and CF tracheas were purchased from Exemplar Genetics and were treated identically. Whenever possible, WT, CFTR ${ }^{+}$,- and CF samples from the same litter were shipped together, but there were enough instances when this was not possible to preclude the use of paired statistics. The interval between time of euthanasia and the start of physiological experiments was approximately 18-24 hours. A total of 21 piglet tracheas was used for these experiments: 9 WT (age, $1.2 \pm 0.2$ days), $2 \mathrm{CFTR}^{+/-}$(age, 1 day), and $10 \mathrm{CF}\left(8 \mathrm{CFTR}^{-/-}, 2 \mathrm{CFTR}^{-/ \triangle F 508}\right.$; age, $1.1 \pm 0.1$ days).

Optical measurement of mucus secretion rates (mucus bubble method). Measurements were made as described previously (14). In brief, a $0.5-\mathrm{cm}^{2}$ piece of mucosa from ventral trachea or bronchus with underlying glands was dissected from the cartilage and mounted at the bath/air interface of a chamber with serosa in the bath ( $\mathrm{pH} 7.4$, with approximately 290 mOsm Krebs-Ringer bicarbonate buffer containing glucose and $1 \mu \mathrm{M}$ indomethacin to minimize prostaglandin release). The tissue surface was cleaned, dried, and layered with approximately $10 \mu \mathrm{l}$ watersaturated mineral oil. Experiments were performed at $37^{\circ} \mathrm{C}$; tissue was superfused with warmed, humidified $95 \% \mathrm{O}_{2}$ and $5 \% \mathrm{CO}_{2}$. Pharmacological agents were diluted to final concentrations with warmed, gassed bath solution and were added to the serosal side by complete bath replacement. Bubbles of mucus within the oil layer were visualized by oblique illumination, and digital images were captured with the macro lens of a Nikon digital camera or by mating a digital camera to one ocular of a Wild stereomicroscope. Stored images were analyzed by direct measurement or with ImageJ software (http://rsb.info.nih.gov/ij/). Mucous volumes were determined from the size of the spherical bubbles. Rates for the indicated drugs were calculated by averaging all secretion rates for each 5-minute interval over the whole exposed period (usually 30 minutes for each drug treatment). This slightly underestimates the true rate of secretion, because it includes an initial period before secretion reaches maximum.
The agonist addition protocol was designed to allow sequential testing of various mediators on the small amounts of tissues available. Considerations included: (a) a sufficient Fsk dose $(3 \mu \mathrm{M})$ to produce reproducible gland stimulation in control and $\mathrm{CFTR}^{+/-}$airways; (b) a synergy paradigm that would make use of the 3- $\mu \mathrm{M}$ Fsk condition as well as a concentration of carbachol that did not produce gland secretion on its own $(0.1 \mu \mathrm{M})$; and (c) a carbachol concentration of 1 instead of $10 \mu \mathrm{M}$ to avoid rapid merging of mucus bubbles with adjacent ones. This was necessary because the density of tracheal glands in piglets is approximately 7-8 times higher than that in adult pigs (15).

Gland density was measured by counting the number of mucus bubbles in a defined area after stimulation, taking care to avoid undercounting of merged bubbles, and expressed as number of glands per square millimeter. 3 areas under the oil-covered trachea were randomly selected $\left(\sim 3 \mathrm{~mm}^{2} /\right.$ trachea) and averaged for 10 control (total $\left.30 \mathrm{~mm}^{2}\right)$ and $9 \mathrm{CF}$ piglets $\left(27 \mathrm{~mm}^{2}\right)$.

Reagents. Compounds (Sigma-Aldrich) were made fresh or maintained at $-20^{\circ} \mathrm{C}$ as aliquots of stock solutions. Stock solutions of SubP, phosphoramidon (a protease inhibitor used together with SubP to prevent potential SubP breakdown), and carbachol were dissolved in sterile distilled water; indomethacin was in ethanol; and Fsk was in DMSO. Drugs in the gland secretion experiments were diluted 1:1,000 with bath solution immediately before use at the indicated concentrations. The highest DMSO concentration in our experiments was $0.1 \%$.

Statistics. Data are shown as mean \pm SEM, and Student's 2-tailed $t$ test for unpaired data was used to compare the means of different treatment groups, unless otherwise indicated. The difference between the 2 means was considered significant for $P$ values less than 0.05 .

\section{Acknowledgments}

We thank Mauri Krouse, Michael Welsh, David K. Meyerholz, David A. Stoltz, and Jin V. Wu for valuable discussions; Jennifer Lyons for suggestions; and Kim V. Tran for technical assistance. This work was supported by NIH grant HL51670 from the National Heart, Lung, and Blood Institute, the Cystic Fibrosis Foundation, and Cystic Fibrosis Research Inc.

Received for publication April 23, 2010, and accepted in revised form June 1, 2010.

Address correspondence to: Jeffrey J. Wine, Cystic Fibrosis Research Laboratory, Room 450, Bldg. 420, Main Quad, Stanford University, Stanford, California 94305-2130, USA. Phone: 650.725.2462; Fax: 650.725.5699; E-mail: wine@stanford.edu.
1. Rowe SM, Miller S, Sorscher EJ. Cystic fibrosis. NEngl J Med. 2005;352(19):1992-2001.

2. Rogers CS, et al. Production of CFTR-null and CFTR-DeltaF508 heterozygous pigs by adeno-associated virus-mediated gene targeting and somatic cell nuclear transfer. J Clin Invest. 2008;118(4):1571-1577.

3. Rogers CS, et al. The porcine lung as a potential model for cystic fibrosis. Am J Physiol Lung Cell Mol Physiol. 2008;295(2):L240-L263.

4. Rogers CS, et al. Disruption of the CFTR gene produces a model of cystic fibrosis in newborn pigs. Science. 2008;321(5897):1837-1841.

5. Stoltz DA, et al. Cystic fibrosis pigs develop lung disease and exhibit defective bacterial eradication at birth. Sci Transl Med. 2010;2(29):29ra31.

6. Joo NS, Irokawa T, Wu JV, Robbins RC, Whyte RI, Wine JJ. Absent secretion to vasoactive intestinal peptide in cystic fibrosis airway glands. J Biol Chem. 2002;277(52):50710-50715.

7. Choi JY, et al. Synergistic airway gland mucus secretion in response to vasoactive intestinal peptide and carbachol is lost in cystic fibrosis. J Clin Invest.
2007;117(10):3118-3127.

8. Choi JY, et al. Substance P stimulates human airway submucosal gland secretion mainly via a CFTR-dependent process. J Clin Invest. 2009; 119(5):1189-2000.

9. Jayaraman S, Joo NS, Reitz B, Wine JJ, Verkman AS. Submucosal gland secretions in airways from cystic fibrosis patients have normal $[\mathrm{Na}+]$ and $\mathrm{pH}$ but elevated viscosity. Proc Natl Acad Sci U S A. 2001;98(14):8119-8123.

10. Wine JJ, Joo NS. Submucosal glands and airway defense. Proceedings of the American Thoracic Society. 2004;1(1):47-53.

11. Salinas D, et al. Submucosal gland dysfunction as a primary defect in cystic fibrosis. FASEB J. 2005;19(3):431-433.

12. Joo NS, Irokawa T, Robbins RC, Wine JJ. Hyposecretion, not hyperabsorption, is the basic defect of cystic fibrosis airway glands. J Biol Chem. 2006;281(11):7392-7398.

13. Meyerholz DK, et al. Loss of CFTR function produces abnormalities in tracheal development in neonatal pigs and young children [published online ahead of print July 9, 2010]. Am J Respir Crit Care Med. doi:10.1164/rccm.201004-0643OC.

14. Joo NS, Wu JV, Krouse ME, Saenz Y, Wine JJ. Optical method for quantifying rates of mucus secretion from single submucosal glands. Am J Physiol Lung Cell Mol Physiol. 2001;281(2):L458-L468.

15. Joo NS, Saenz Y, Krouse ME, Wine JJ. Mucus secretion from single submucosal glands of pig. Stimulation by carbachol and vasoactive intestinal peptide. J Biol Chem. 2002;277(31):28167-28175.

16. Trout L, Corboz MR, Ballard ST. Mechanism of substance P-induced liquid secretion across bronchial epithelium. Am J Physiol Lung Cell Mol Physiol. 2001;281(3):L639-L645.

17. Lamb JP, Sparrow MP. Three-dimensional mapping of sensory innervation with substance $\mathrm{p}$ in porcine bronchial mucosa: comparison with human airways. Am J Respir Crit Care Med. 2002;166(9):1269-1281.

18. Berschneider HM, et al. Altered intestinal chloride transport in cystic fibrosis. FASEB J. 1988; 2(10):2625-2629. 
19. Taylor CJ, Baxter PS, Hardcastle J, Hardcastle PT. Failure to induce secretion in jejunal biopsies from children with cystic fibrosis. Gut. 1988;29(7):957-962.

20. Bijman J, et al. Chloride transport in the cystic fibrosis enterocyte. Adv Exp Med Biol. 1991;290:287-294.

21. Ousingsawat J, Martins JR, Schreiber R, Rock JR, Harfe BD, Kunzelmann K. Loss of TMEM16A causes a defect in epithelial Ca2+ dependent chloride transport. J Biol Chem. 2009;284(42):28698-28703.

22. Lee RJ, Foskett JK. Mechanisms of Ca2+-stimulated fluid secretion by porcine bronchial submucosal gland serous acinar cells. Am J Physiol Lung Cell Mol Physiol. 2009;298(2):L210-L231.

23. Boucher RC. Airway surface dehydration in cystic fibrosis: pathogenesis and therapy. Annu Rev Med. 2007;58:157-170.

24. Ianowski JP, Choi JY, Wine JJ, Hanrahan JW. Mucus secretion by single tracheal submucosal glands from normal and cystic fibrosis transmembrane conductance regulator CFTR knock-out mice. JPhysiol. 2007;580(pt 1):301-314.

25. Wine JJ. Parasympathetic control of airway submucosal glands: Central reflexes and the airway intrinsic nervous system. Auton Neurosci. 2007;133(1):35-54.

26. Ballard ST, Spadafora D. Fluid secretion by submucosal glands of the tracheobronchial airways. Respir Physiol Neurobiol. 2007;159(3):271-277.

27. Joo NS, Lee DJ, Winges KM, Rustagi A, Wine JJ.
Regulation of antiprotease and antimicrobial protein secretion by airway submucosal gland serous cells. J Biol Chem. 2004;279(37):38854-38860.

28. Clarke LL, et al. Abnormal Paneth cell granule dissolution and compromised resistance to bacterial colonization in the intestine of CF mice. Am J Physiol Gastrointest Liver Physiol. 2004;286(6):G1050-G1058.

29. Conner GE, Wijkstrom-Frei C, Randell SH, Fernandez VE, Salathe M. The lactoperoxidase system links anion transport to host defense in cystic fibrosis. FEBS Lett. 2007;581(2):271-278.

30. Moskwa P, et al. A novel host defense system of airways is defective in cystic fibrosis. Am J Respir Crit Care Med. 2006;175(2):174-183. 